\title{
Traffic Avoidance and Separation System
}

\author{
Balaji Raman Katta \\ Strategic Electronics R\&D Centre \\ Hindustan Aeronautics Limited \\ Hyderabad, India \\ balaji.raman@hal-india.com
}

\author{
Irfan Madani \\ School of Aerospace, Transport \& Manufacturing \\ Cranfield University, \\ Cranfield, United Kingdom \\ k.i.madani@cranfield.ac.uk
}

\begin{abstract}
Aircraft need to be separated from other aircraft by either a minimum vertical distance of $1000 \mathrm{ft}$ or by a minimum horizontal distance of $5 \mathrm{NM}$ to avoid mid-air collisions. Traffic Alert and Collision Avoidance System (TCAS) work well as a tactical safety backups to avoid collision, but cannot perform separation assurance. This paper presents the design aspects of system that would predict separation infringement and provide flight crew with necessary guidance for maintaining separation. This paper discusses formulation of system function based on ADS-B, mathematical models, simulation and results. A system model has been implemented in MATLAB and various encounters with different speed ratios at various conflict angles has been fed as input to system model with a goal of avoidance and recovery to original waypoint. Both Heading and Speed maneuvers are evaluated and results are presented. The required strength of resolution maneuver as a function of conflict geometry is studied and automatic maneuver selection function is implemented in system model and maneuver selection function is evaluated with discussion of results.
\end{abstract}

Keywords-Free Flight, ASAS, ACAS, CD\&R, ADS-B, ATM, Self-Separation.

\section{INTRODUCTION}

Aircraft need to be separated from other aircraft by either a minimum vertical distance of $1000 \mathrm{ft}$ or by a minimum horizontal distance of $5 \mathrm{NM}$ to avoid mid-air collisions. In the current air traffic management (ATM) the primary responsibility of separation assurance function lies with the ground controller while pilots have limited role. Next generation ATM aims to achieve increase in airspace capacity and improve safety factor. With steady increase in air traffic, current air traffic control (ATC) may not handle proper separation assurance [1]. Traffic alert and collision avoidance system (TCAS) work well as a tactical safety backups to avoid collision, but cannot perform separation assurance [2]. Future ATM concept envisages delegation of separation assurance responsibility to flight crew either completely or partially with help of an on-board advisory system which would maintain standard separation which will be called as separation assurance (SA) system [3]. Unlike TCAS, separation system can predict and provide resolution guidance to avoid a conflict even in the form of heading control and speed control apart from altitude control. The enabler of this technology is Automatic Dependent Surveillance-Broadcast (ADS-B).
This paper presents ADS-B concept, realization of separation assurance function based on ADS-B. The purpose of this study is to simulate and analyze the behavior of conflict resolution maneuver as function of conflict geometry. Section-II introduces ADS-B surveillance concept, realization of separation assurance function based on ADS-B and discussion of conflict zones. Section-III presents the mathematical formulation of separation infringement detection and resolution algorithm. Section-IV presents the experiment and simulation setup of separation assurance system with discussion of results.

TABLE I.

\begin{tabular}{|l|l|c|}
\hline Sl.No & \multicolumn{1}{|c|}{ Report } & $\begin{array}{c}\text { Information } \\
\text { Elements }\end{array}$ \\
\hline 1 & State Vector Report & $\begin{array}{c}\text { 3D position, 3D Velocity, } \\
\text { Navigation Integrity }\end{array}$ \\
\hline 2 & Mode Status Report & $\begin{array}{c}\text { Call Sign, Aircraft length \& } \\
\text { Width codes, Navigation } \\
\text { Accuracy for position and } \\
\text { Velocity }\end{array}$ \\
\hline 3 & Target State Report & Selected Altitude and Heading \\
\hline 4 & Trajectory Change Point & TCP, TCP+1 \\
\hline
\end{tabular}

\section{CONFLICT ZONES AND SEPARATION ASSURANCE}

ADS-B is an aircraft function that enables periodic transmission of state vector identification, Mode status information and other information as listed in table-I. ADS-B enhances pilot's situational awareness and safety. ADS-B provides accurate surveillance by combining GPS source, and ground ATC. ADS-B is an important element of future ATM that will shift air traffic control (ATC) from radar to a satellite based system [4]. ADS-B provides wide information about aircraft state, intent and identification etc in the form of reports which can be used for maintaining separation.

SA system uses the ADS-B reports to acquire information about surrounding target and assess the information for any potential conflicts and provides necessary resolution guidance to avoid conflicts. Three dimensional position information received in state vector report is used to calculate horizontal and vertical separation between participating aircraft. The current three dimensional velocity information when combined with current position can be used to predict future positions of 


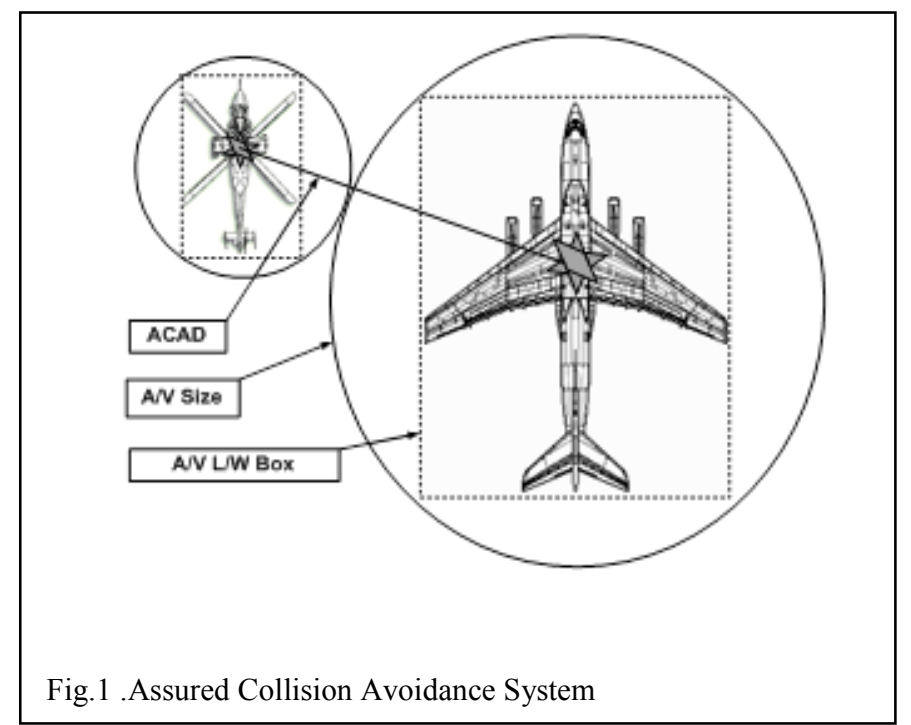

aircraft within a given look-ahead time. These predicted positions can be used to know whether aircraft violate $5 \mathrm{NM}$, $1000 \mathrm{ft}$ separation in future. Mode status report consists of aircraft length \& width codes, and navigation uncertainties (NACp, NACv, SIL). Aircraft length and width codes can be used to obtain the physical space occupied by each aircraft and this can be used to derive minimum distance required to avoid a collision. While performing separation assurance or collision avoidance, respective functions should also cater for position and velocity uncertainties which can be deduced from NACp, $\mathrm{NACv}$ fields. Conflict detection involves prediction based on present and past state vector information which has inherent error.

Extrapolation of position based on state vector information would result in large errors with longer look-ahead times for conflict detection and resolution for separation assurance [5]. The state vector information can be augmented with intent information like trajectory change points, target state etc. The knowledge of planned changes to the current path is required for efficient conflict management like avoiding false alerts and strategic re-routing in the event of predicted conflict [5]. Target state report consists of intent information like selected altitude and heading while trajectory change report consists of current and future trajectory change point information. Intent information is provided to increase efficiency of trajectory prediction in conflict detection and resolution, in a way it allows longer look-ahead times. Target altitude is the desired flight level for level off during climb or descent or the desired altitude during level flight. Desired track is the intended final track during a horizontal direction or the track to be maintained during normal flight. When aircraft are maneuvering, its final state can be deduced from this tactical intent in selected altitude and mode indication information.

Collision Avoidance Zone (CAZ) is a protected airspace around each aircraft, when entered by another aircraft would result in a collision or near miss. The CAZ is made of three components assured collision avoidance distance, position

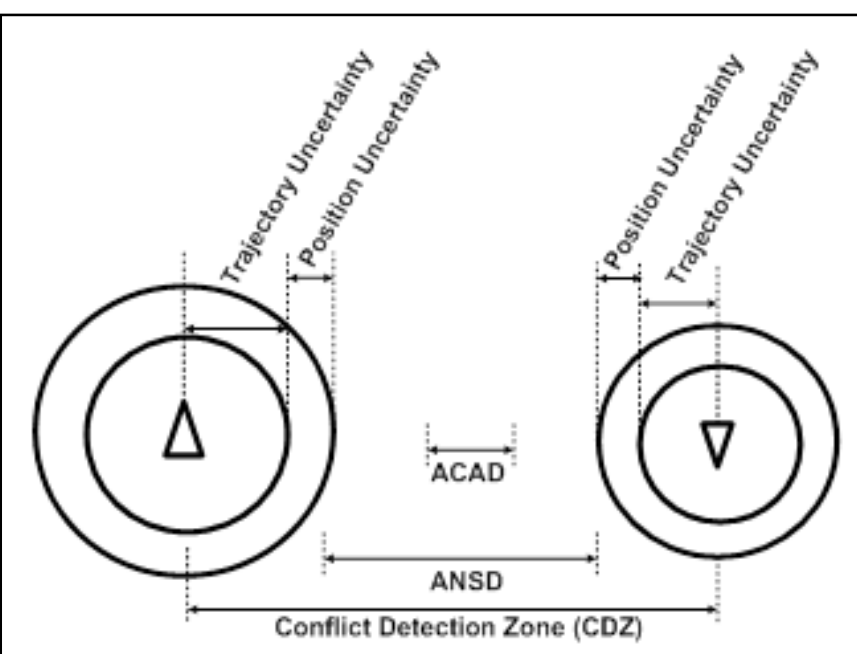

Fig.2. Conflict Detection Zone (CDZ)

and trajectory uncertainty as shown in Fig.2 [6]. The assured collision avoidance distance (ACAD) is minimum distance that is needed to avoid collision which depends on the size of the aircrafts in conflict as shown in Fig.1 [7]. TCAS generates alerts in the form of vertical speed change when CAZ is penetrated by other aircraft with a look-ahead time 30-40 sec. Adding both the uncertainties of each aircraft with ACAD results in a distance that is sufficient to avoid a near miss/ collision, even in presence of position error and flight technical error.

A 5nmi horizontal and $1000 \mathrm{ft}$ vertical separation is used in enroute flight, while a $3 \mathrm{nmi}$ horizontal and $1000 \mathrm{ft}$ vertical separation is used in terminal area[8]. As per RTCA DO-289, required horizontal separation minima are also known as assured nominal separation distance (ANSD). Conflict detection zone is a wider protected airspace around each aircraft, when entered by other aircraft would result in violation of legal separation between aircraft. CDZ is similar to CAZ except that it is a combination of position uncertainty, trajectory uncertainty and ANSD as shown in Fig.2 [6]. Separation assurance system builds conflict detection zone based on NACp, NACv values. Then system utilizes the ADS$B$ state vector and Intent information to predict and avoid separation infringement by providing alerts and guidance to flight crew when $\mathrm{CDZ}$ is penetrated by other aircraft. The typical look-ahead time is between 5-10 minutes. The system is capable of providing resolution manoeuvre in horizontal plane in the form of commanded heading and commanded speed for conflict avoidance.

\section{SA SYSTEM DESIGN}

The separation infringement detection and resolution utilised by SA system proposed in this paper based on Geometric CD\&R method as proposed in Ref [9]. To make clear distinction between conflict detection and resolution used for tactical system like TCAS, the CD\&R used for separation assurance in this paper will be referred to as Separation Infringement Detection and Resolution (SIDAR). 


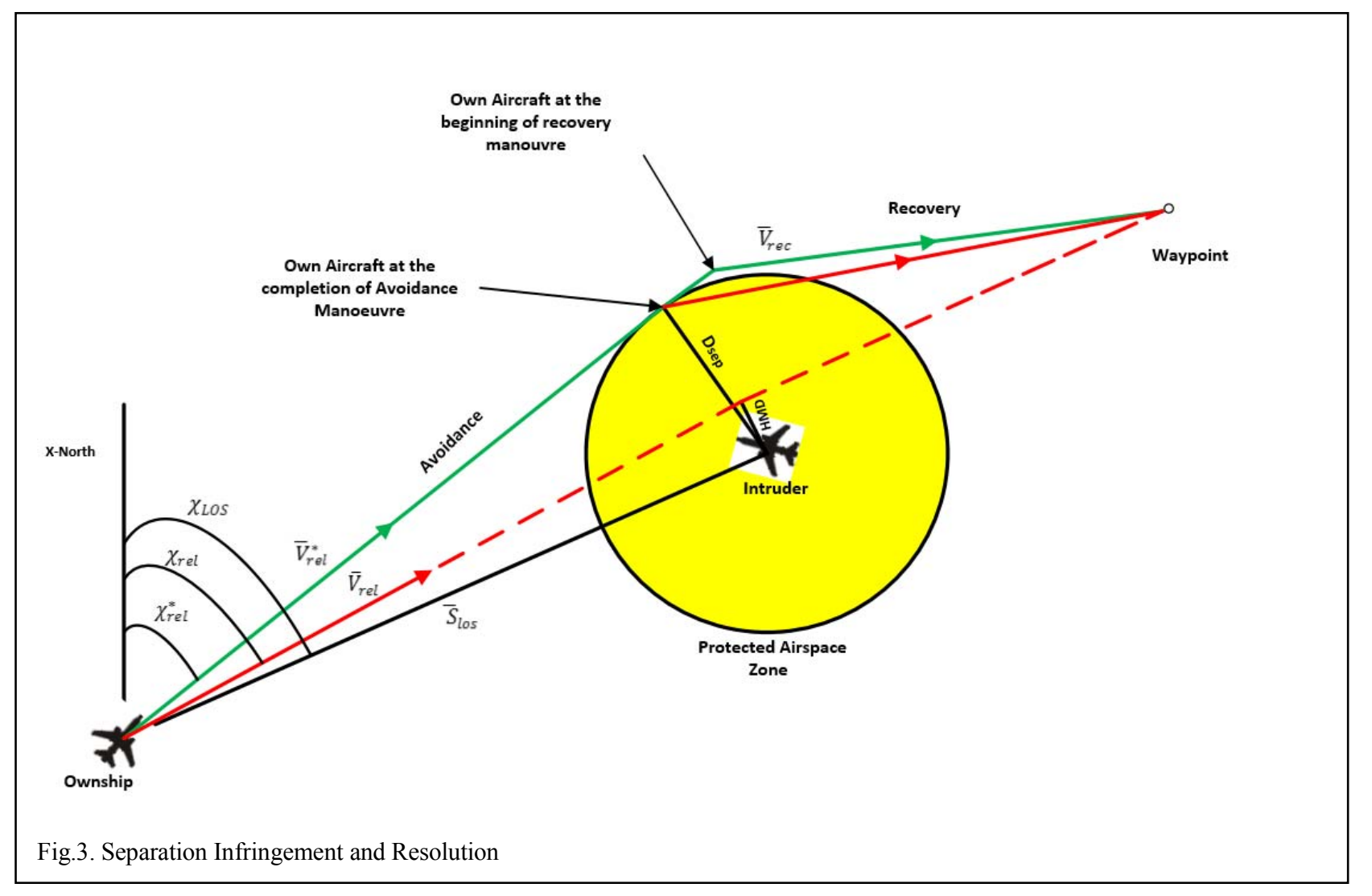

\section{A. Infringement Detection Algorithm}

Let $\bar{S}_{o}\left(x_{o}, y_{o}\right)$ and $S_{i}\left(x_{i}, y_{i}\right)$ be the 2D position coordinate vectors of own aircraft (O) and intruder aircraft (I) respectively to an earth fixed axis system defined with $\mathrm{x}$ - axis pointing north and y-axis pointing east as shown in Fig. 3 . Let $\bar{V}_{o}$ and $\bar{V}_{i}$ are the velocity vectors of own aircraft $(\mathrm{O})$ and intruder aircraft (I) in the horizontal plane. Let $V_{o}$ and $V_{i}$ represent the corresponding ground speeds and $\chi_{o}$ and $\chi_{i}$ are the corresponding heading angles measured clockwise from the $\mathrm{x}$-axis pointing north. Assuming constant velocity the horizontal positions of own and intruder aircraft at a time $t \geq 0$ are given by

$$
\begin{aligned}
& \bar{S}_{o}(t) \equiv \bar{S}_{o}+t \bar{V}_{o} \\
& \bar{S}_{i}(t) \equiv \bar{S}_{i}+t \bar{V}_{i}
\end{aligned}
$$

A relative coordinate system is used for mathematical simplification where the one aircraft is at the centre of the system with intruder located at relative position vector $\bar{S}_{l o s}$ as shown in Fig. 3. It is also known as line of sight vector (LOS) vector from own aircraft $(\mathrm{O})$ to intruder aircraft (I). The relative position vector, its magnitude $S_{l o s}$ and azimuth angle $\chi_{\text {los }}$ are given by

$$
\begin{gathered}
\bar{S}_{l o s}=\left(S_{o}-S_{i}\right) \\
S_{l o s}=\sqrt{\left(x_{o}-x_{i}\right)^{2}+\left(y_{o}-y_{i}\right)^{2}}
\end{gathered}
$$

$$
\chi_{\text {los }}=\tan ^{-1}\left(\frac{y_{o}-y_{i}}{x_{o}-x_{i}}\right)
$$

The magnitude $V_{\text {rel }}$ and azimuth angle $\chi_{\text {rel }}$ of the relative velocity vector are given by:

$$
\begin{gathered}
\bar{V}_{r e l}=\left(\bar{V}_{o}-\bar{V}_{i}\right) \\
V_{r e l}=\sqrt{V_{o}^{2}+V_{i}^{2}+2 V_{o} V_{i} \cos \left(\chi_{o}-\chi_{i}\right)} \\
\chi_{r e l}=\tan ^{-1}\left(\frac{V_{o} \sin \chi_{o}-V_{i} \sin \chi_{i}}{V_{o} \cos \chi_{o}-V_{i} \cos \chi_{i}}\right)
\end{gathered}
$$

The relative horizontal position vector of Intruder with respect to own aircraft at any time can be defined by:

$$
\bar{S}_{l o s}(t) \equiv \bar{S}_{l o s}+t \bar{V}_{r e l}
$$

The time to closest point of approach (CPA), $\tau_{C P A}$ can be found by finding the value of time for which the separation distance achieves a minimum.

$$
\begin{gathered}
\frac{d}{d t}\left(\bar{S}_{l o s}+t \bar{V}_{r e l}\right) *\left(\bar{S}_{l o s}+t \bar{V}_{r e l}\right)=0 \\
\tau_{C P A}=-\frac{\bar{S}_{l o s} \cdot \bar{V}_{r e l}}{\left\|V_{r e l}\right\|^{2}}
\end{gathered}
$$

The closest approach distance or horizontal miss distance is given by

$$
H M D \equiv \bar{S}_{\text {los }}+\tau_{C P A} \bar{V}_{\text {rel }}
$$

Let $D_{\text {sep }}$ be the horizontal separation minima, the horizontal miss distance, should be greater than separation standard 
$H M D>D_{\text {sep }}$. The dot product of line of sight vector $\bar{S}_{l o s}$ and relative velocity vector $\bar{V}_{r e l}$ also characterizes whether the aircraft are horizontally diverging i.e. $\bar{S}_{l o s} \cdot \bar{V}_{r e l}>0$ horizontally converging i.e. $\bar{S}_{l o s} \cdot \bar{V}_{r e l}<0$. A separation infringement is predicted to occur, if it satisfies the following condition within a specified look-ahead time.

$$
H M D<D_{\text {sep }} \text { and } \bar{S}_{\text {los }} \cdot \bar{V}_{\text {rel }}<0
$$

\section{B. Infringement Resolution Algorithm}

In order to resolve the separation infringement the direction of the relative velocity vector $\bar{V}_{r e l}$ has to be changed so that it's the predicted state of the aircraft does not pass through the protection zone of the other aircraft as shown in Fig. 3. This can be achieved either by changing the direction i.e. the heading angle and /or the magnitude i.e. the speed of the ownship velocity vector $\bar{V}_{o}$ and/or intruder velocity vector $\bar{V}_{i}$. Let $\bar{V}_{\text {rel }}^{*}$ represent the new relative avoidance velocity vector and $\chi_{\text {rel }}^{*}$ be the new azimuth angle. The separation infringement can be resolved if the azimuth of the relative velocity vector is changed from $\chi_{\text {rel }}$ to $\chi_{\text {rel }}^{*}$ which can be determined from the geometry in the Fig. 3, by following equation.

$$
\chi_{\text {rel }}^{*}=\chi_{\text {rel }} \pm \sin ^{-1}\left(\frac{D_{\text {sep }}}{S_{\text {los }}}\right)
$$

Let own aircraft contributes to resolution by changing the azimuth angle of the relative velocity vector from $\chi_{\text {rel }}$ to $\chi_{\text {rel }}^{*}$. Let $\bar{V}_{o}^{*}$ be the own aircraft's new velocity vector, the magnitude and azimuth angle of this vector be denoted by $V_{o}^{*}$ and $\chi_{o}^{*}$ respectively. For a conflict resolution, with heading change alone, the new heading angle can be obtained from equation below [3].

$$
\chi_{o}^{H C}=\chi_{\text {relo }}^{*}-\sin ^{-1}\left(\frac{V_{i}}{V_{o}} \sin \left(\chi_{\text {relo }}^{*}-\chi_{i}\right)\right)
$$

For a conflict resolution with speed change alone, the new speed can be obtained from equation-(16).
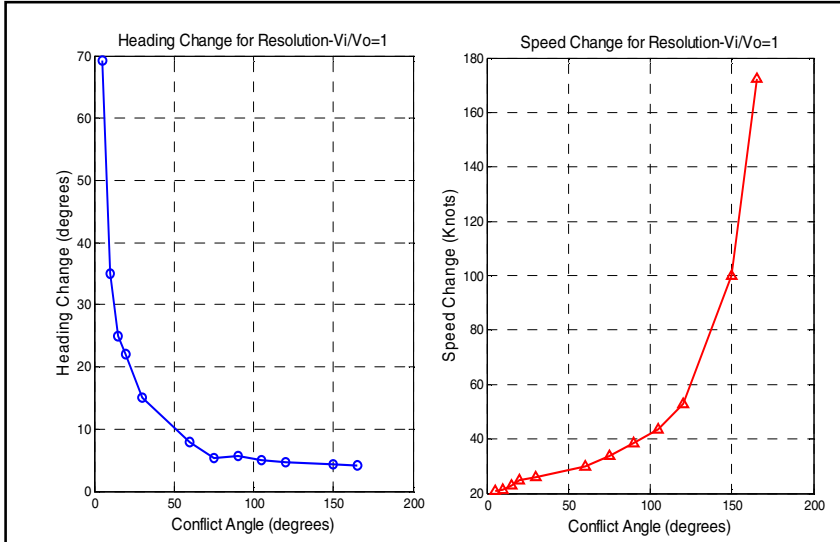

Fig.4. Resolution Maneuver with a Speed Ratio of $\mathrm{V}_{\mathrm{i}} / \mathrm{V}_{\mathrm{o}}=1$

$$
V_{o}^{S C}=V_{i}\left(\frac{\sin \left(\chi_{\text {relo }}^{*}-\chi_{i}\right)}{\sin \left(\chi_{\text {relo }}^{*}-\chi_{o}\right)}\right)
$$

The resulting values of $\mathbb{7}_{0}^{S E}$ is valid only if it lies within the bounds of aircraft performance. If the desired solution is not valid, second solution can be used. If both speeds are not valid, then conflict can be resolved by heading change.

\section{SIMULATION AND RESULTS}

SIMULINK model is developed to test and verify tactical intent based separation assurance function for medium term conflicts with $10 \mathrm{~min}$ look-ahead time. This block is based on mathematical models discussed in section III of this paper.

\section{A. Effect of Conflict Angle on Manouvre Strength}

The effect of conflict angle on the magnitude of speed and heading manouvres is studied at various speed ratios. In order to resolve the separation infringement the direction of the relative velocity vector $\bar{V}_{\text {rel }}$ has to be changed either by changing the heading angle or the speed of the ownship velocity vector $\bar{V}_{o}$ and/or intruder velocity vector $\bar{V}_{i}$. Paper considers changing only ownship velocity vector characterstics as it is desirable to change one aircraft flight path instead of both aircraft, from safety point of view. The relative vector $\bar{V}_{r e l}$ has to be rotated by an angle for resolution, either by heading or speed change by ownship as shown in Fig-3, this would in turn cause change in magnitude of relative velocity vector defined by equation-(7). For a given rotation of relative vector, the manouvre that causes minimum change in magnitude of relative vector would be considered as optimal maneuver. The simulation is conducted with two aircraft flying at different speed ratios $\left(V_{o} / V_{i}\right)$ and their initial positions are such that they meet the intersection point (collision point) at same time. The $5 \mathrm{nmi}$ horizontal separation is used which is the sum of $3 \mathrm{nmi}$ assured nominal separation distance (ANSD) and $2 \mathrm{nmi}$ position uncertainty of either aircraft. For each speed ratio, the intruder aircraft flies on 180 constant heading and the own aircraft heading is varied from $195^{\circ}$ to $360^{\circ}$ degrees to vary the the conflcit angle from $5^{\circ}$ degrees to $180^{\circ}$ degrees. This is to study how the required heading and speed maonouvre strength varies with

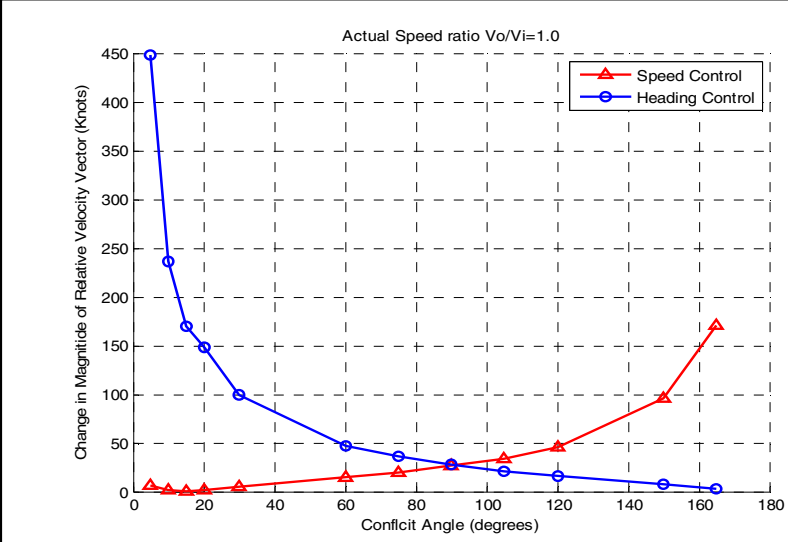

Fig.5. Change in Magnitude of Relative Velocity Vector 

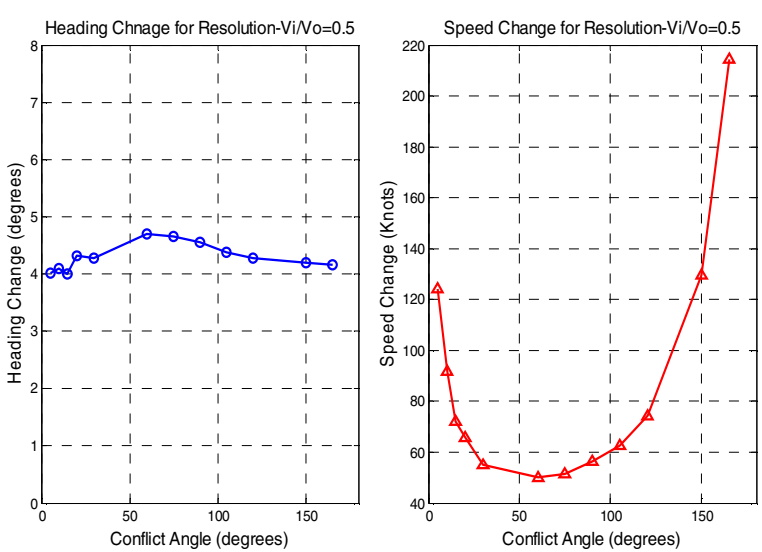

Fig.6. Resolution Maneuver with a Speed Ratio of $\mathrm{V}_{\mathrm{i}} / \mathrm{V}_{\mathrm{o}}=0.5$

conflict angle at different speed ratios and also to find the best optimal maneuver. Only backside solutions i.e. heading increase and speed decrease manouvres are considered where manouvering ownship passes behind the intruder. However the same exeperiment can also be conducted for frontside soultion where the manouvering ownship passes ahead of intruder.

\section{1) Manouvre Strength when Speed Ratio $V_{i} / V_{o}=1$}

In this scenario, ownship speed is 400 knots and intruder speed is 400 knots. From Fig-4, as the conflict angle increases the magnitude of heading control manoeuvres decreases. The required heading change at smaller conflict angle is large and decreases with increase in conflict angle. It is important to confirm that magnitude of the heading manoeuvre is within the aircraft's maximum turn rate capability, before executing the manoeuvre. Considering a maximum heading change limit of $17^{\circ}$, conflict resolution is possible between conflict angles $25^{\circ}$ to $180^{\circ}$. The change in magnitude of relative velocity vector due to heading change decreases with increase in conflcit angle.

On the other hand, speed control exhibits completely opposite
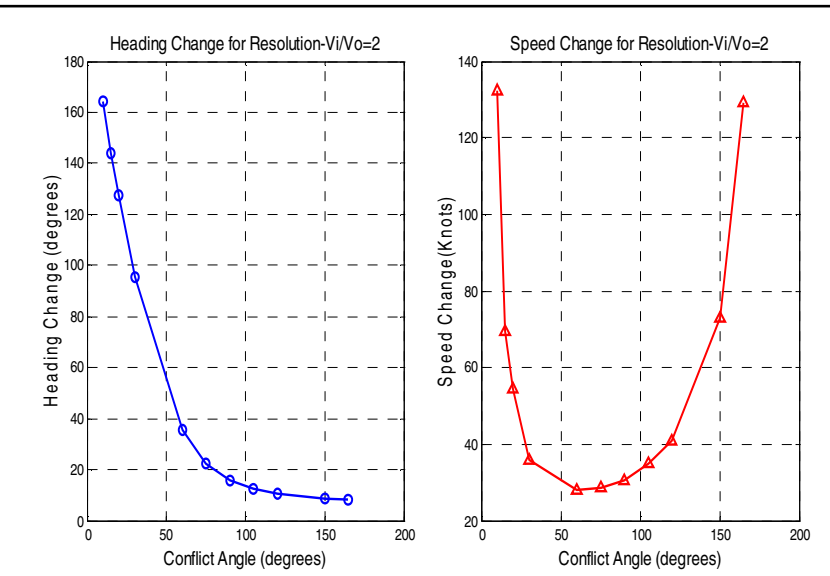

Fig.8. Resolution Maneuver with a Speed Ratio of $\mathrm{V}_{\mathrm{i}} / \mathrm{V}_{\mathrm{o}}=2$

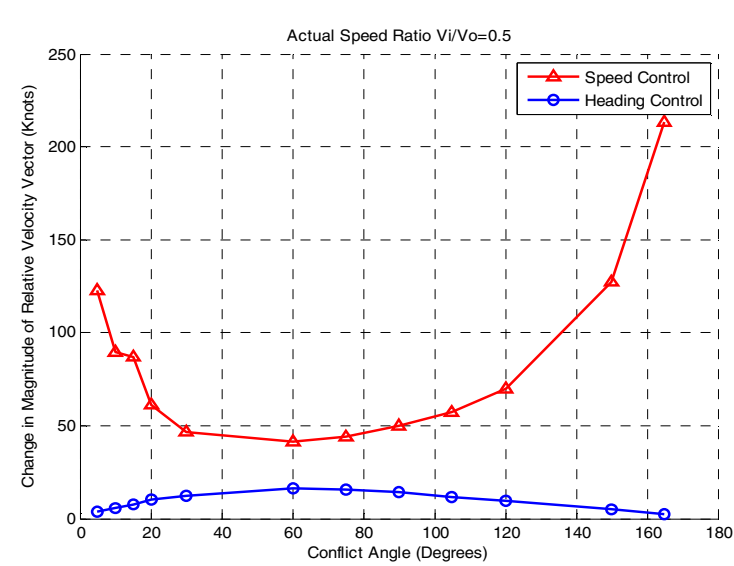

Fig.7. Change in Magnitude of Relative Velocity Vector

behaviour to that of heading control. As the conflict increases the magnitude of speed control manoeuvres increases. The required speed change at smaller conflict angle is small and increases with increase in conflict angle. It is important to confirm that magnitude of the speed maneuver is within the aircraft's speed capability, before executing the manoeuvre. The solution not within aircraft performance limits will be discarded. As seen from Fig. 5, the change in magnitude of relative velocity vector due to speed change increases with increase in conflcit angle. At conflcit angles above $90^{\circ}$, the change in magnitude of relative velocity vector due to heading change is less than that of speed change. At conflcit angles below $90^{\circ}$ the change in magnitude of relative velocity vector due to speed change is less than that of heading change. So at higher conflict angles $\left(>90^{\circ}\right)$ heading manouvre is optimal while speed is optimal at at lower conflcit angles $\left(<90^{\circ}\right)$. The decisive conflict angle for this speed ratio is $90^{\circ}$ which shall be used for manouvre selection.

\section{2) Manouvre Strength when Speed Ratio $V_{i} / V_{o}=0.5$}

In this scenario, ownship speed is 400 knots and intruder speed is 200 knots. From Fig. 6, the speed resolution is minimum at a conflict angle of $60^{\circ}$. The required speed change

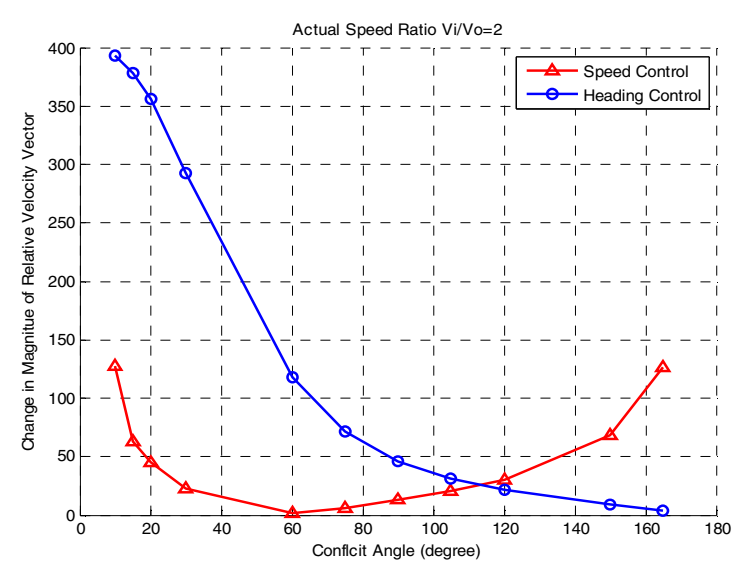

Fig.9.Change in Magnitude of Relative Velocity Vector 

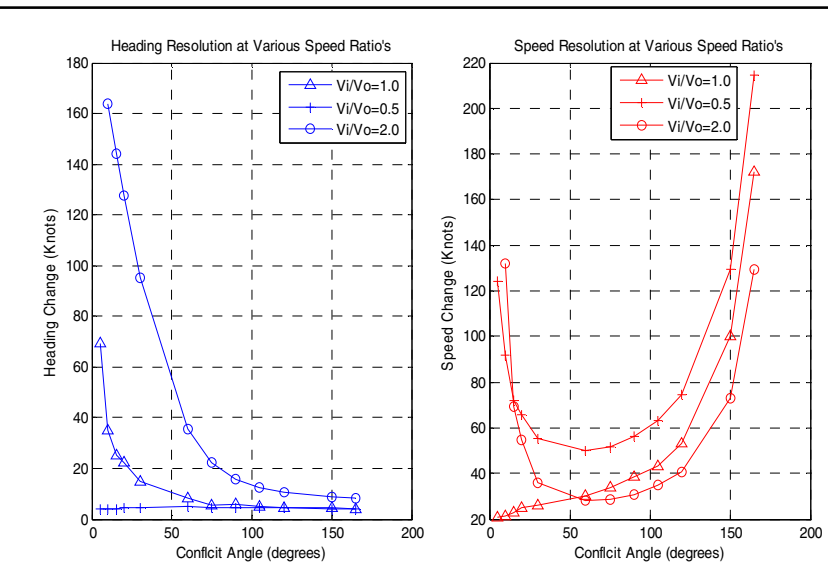

Fig.10. Comparison of maneuvers at various speed ratio's

increases as the angle is either increased or decreased from $60^{\circ}$. Considering the aircraft performance limit of 100 knots for decreasing the speed, the conflict resolution is possible between conflict angles $10^{\circ}$ to $135^{\circ}$. The Heading change required for conflict resolution at this speed ratio is almost constant at around $4^{\circ}$, hence heading maneuver can resolve the conflict between angles from $0^{\circ}$ to $180^{\circ}$. The Fig.7, presents change in magnitude of relative velocity vector due to heading and speed change maneuvers. The change in magnitude due to speed control is minimum with a value of 40 knots at $60^{\circ}$ angle and it increases on either side of $60^{\circ}$ conflict angle. The change in magnitude of relative velocity vector with heading control is less than that of speed control at all conflict angles with a peak value of 16.35 knots at $60^{\circ}$ angle. Heading control is the optimal maneuver with a speed ratio of $V i / V o=0.5$ at all conflict angles.

\section{3) Manouvre Strength when Speed Ratio $V_{i} / V_{o}=2.0$}

In this scenario, ownship speed is 200 knots and intruder speed is 400 knots. From Fig. 8 speed resolution is minimum (30 knots) at a conflict angle of $60^{\circ}$. The required speed change increases as the angle is either increased or decreased from $60^{\circ}$. The required heading change at smaller conflict angle is large and decreases with increase in conflict angle. Behavior of maneuver strength for this speed ratio is similar to the behavior exhibited at speed ratio of ' 1 '. The change in magnitude of relative velocity vector due to heading and speed change is shown in Fig. 9. Both curves intersect at $115^{\circ}$ conflcit angle. At conflict angles greater than $115^{\circ}$, heading control is optimal manouvre and at lower conflict angles $\left(<115^{\circ}\right)$ speed control is best choice for resolution at speed ratio of $V_{i} / V_{o}=2.0$

\section{4) Comparison of manouvre at various speed ratio's}

From Fig. 10, at a given conflict angle, as the speed ratio increases, the heading change required for resolution increases. This is particularly predominant at lower conflict angles where the required heading change is higher at higher speed ratios. Speed change exhibits a complementary behavior to that of heading change. As the speed ratio increases the strength of speed change required for resolution decreases at given conflict angle.

Considering a heading limit of $17^{\circ}$, as the speed ratio increases, the conflict angles over which conflict is successfully resolved by heading resolution becomes narrower. For example at a speed ratio of $V_{o} / V_{i}=0.5$ resolution is possible at all conflict angles. At speed ratio of $V_{o} / V_{i}=1.0$, resolution is possible between $25^{\circ}$ to $180^{\circ}$ conflict angles. At speed ratio of $V_{o} / V_{i}=2.0$, resolution is possible between $88^{\circ}$ to $180^{\circ}$ conflict angles.

Considering a speed decrease limit of 100 knots, as the speed ratio increases, the conflict angles over which conflict is successfully resolved by speed resolution becomes wider. For example at a speed ratio of $\mathrm{V}_{\mathrm{o}} / \mathrm{V}_{\mathrm{i}}=0.5$, resolution is possible between $10^{\circ}$ to $135^{\circ}$ conflict angles while at speed ratio resolution is possible between $15^{\circ}$ to $160^{\circ}$ conflict angles.

The separation assurance of $5 \mathrm{nmi}$ by heading and speed control are also shown in Fig.11-14 in appendix. As shown in Fig.11-14, ownship avoids loss of separation by controlling heading and speed along with recovery to flight path after clearing conflict. To ensure $5 \mathrm{nmi}$ separation, the protection zones of either aircraft with $2.5 \mathrm{nmi}$ radius should not overlap. Intruder and ownship at four different intervals on either side closest point of approach are shown, where the protection zones of either aircraft do not overlap as they cross each other with heading control and speed control which proves that SA function has been realized.

\section{CONCLUSION AND FURTHER WORK}

The paper presented the formulation of traffic avoidance and separation system based on ADS-B. The behavior of resolution maneuver strength, magnitude of relative velocity vector has been studied with respect to conflict angle and speed ratio which would be helpful in selecting the optimal maneuver depending on the conflict geometry. At large speed ratio's $\left(V_{i} / V_{o} \geq 1.0\right)$ heading control is optimal at large conflict angle while speed control is optimal at small conflict angle. At small speed ratio $\left(V_{i} / V_{o}=0.5\right)$ the heading control is optimal at all conflict angles than speed control.

The simulation assumes that the aircraft state is known perfectly and state extrapolation for prediction occurs without error. However errors propagate with larger look-ahead times, this study can be extended to study the effects of uncertainties by including position and velocity errors. The paper focuses separation infringement and resolution in horizontal plane only using 2D-Geometric CD\&R, this can be extended to the vertical plane by using 3D-Geometric CD\&R. 


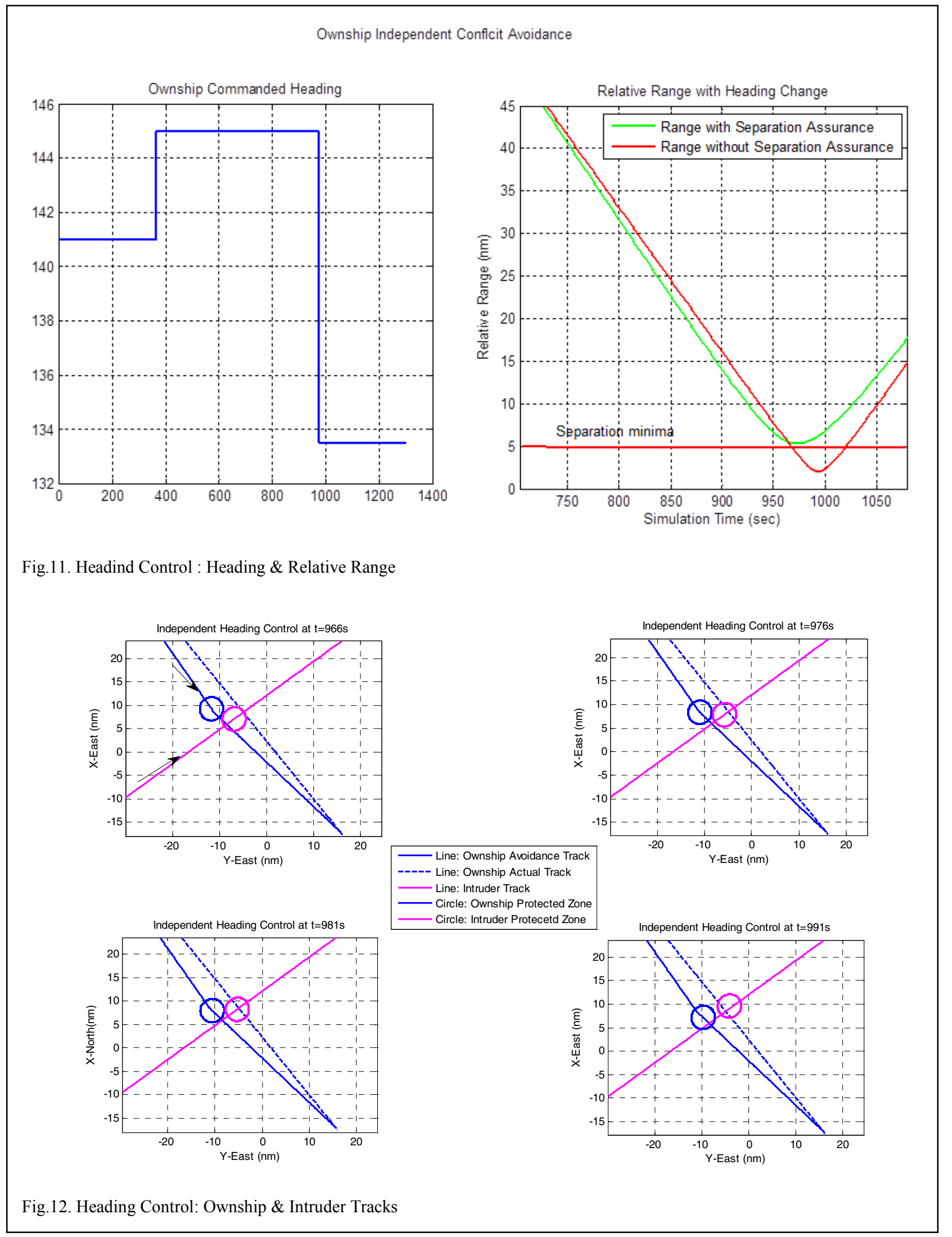



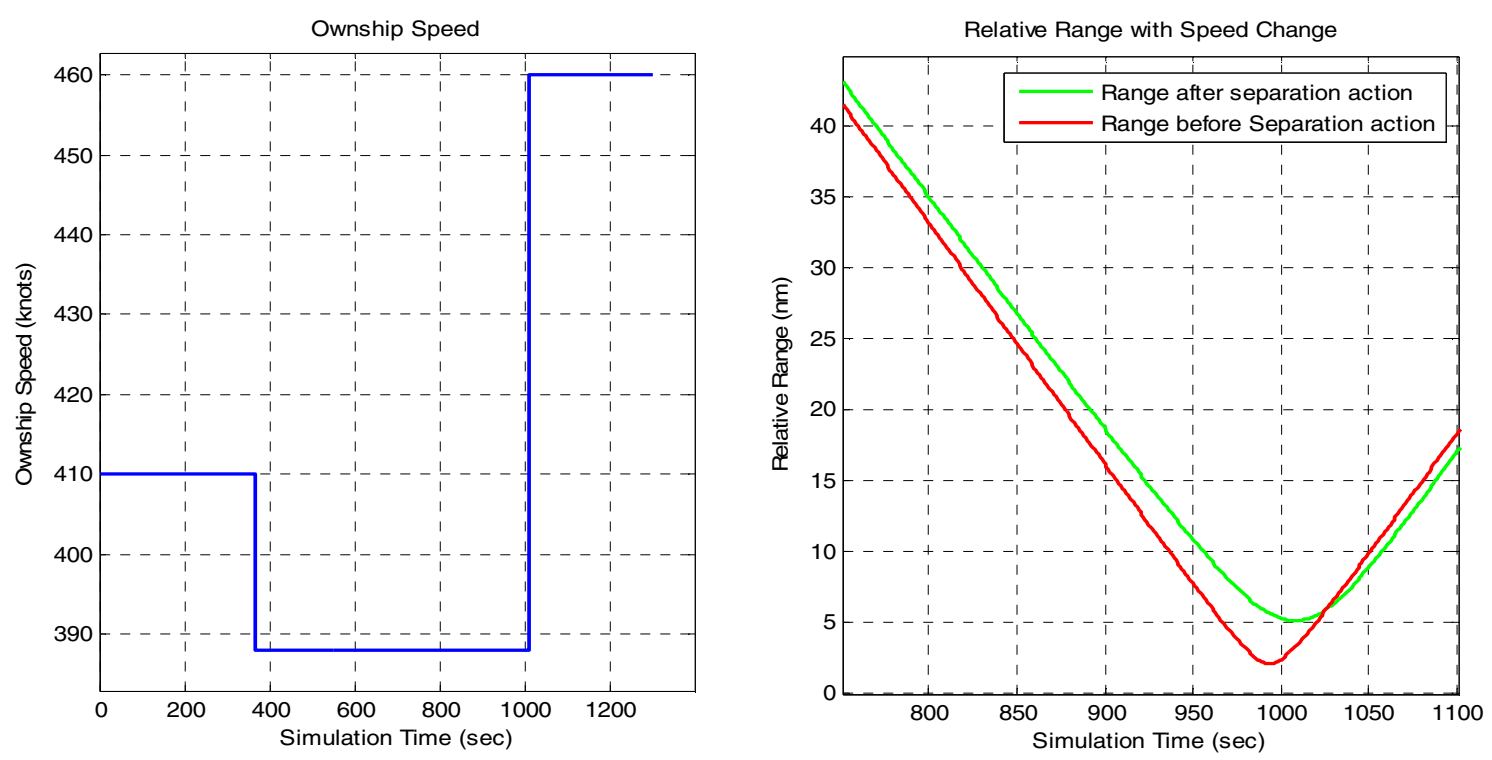

Fig.13. Speed Control : Speed \& Relative Range
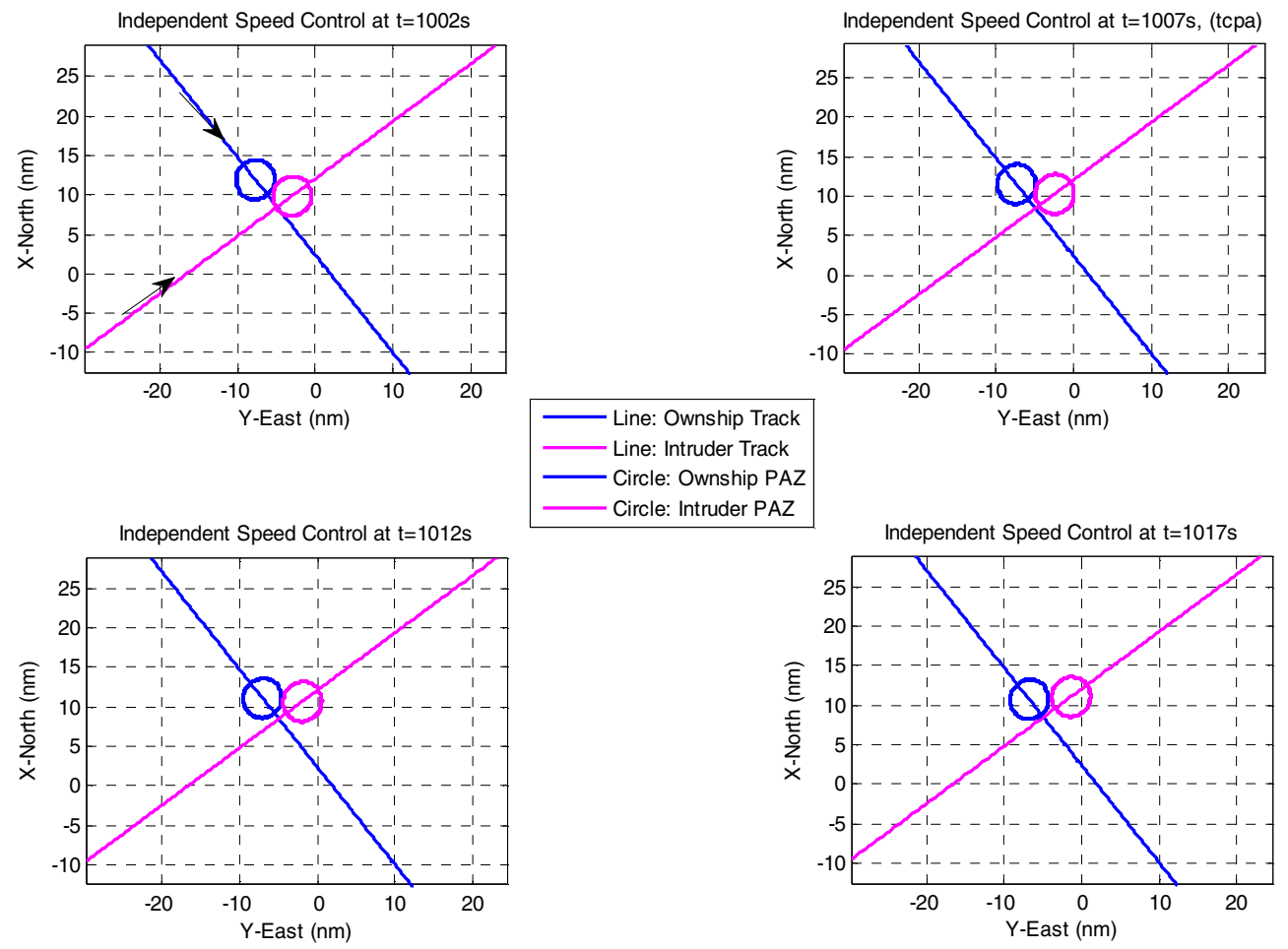

Fig.14. Speed Control: Ownship \& Intruder Tracks 


\section{ACKNOWLEDGEMENTS}

The first author would like to thank Hindustan Aeronautics Limited for MSc sponsorship to Cranfield University, UK.

\section{REFERENCES}

[1] Prevot, T., Homola, J., Mercer, J. (2008), "Human in the Loop Evaluation of Ground-Based Automated Separation Assurance for NextGen," ICAS 2008- 8.4.5, AIAA Aviation Technology Integration and Operations Conference, Anchorage, Alaska, Sept 15-19, 2008.

[2] Dwyer JP and Landry SJ. Separation assurance and collision avoidance concepts for the next generation air transportation system. In: Proceedings of symposium on human interface 2009, San Diego, CA, USA, 19-24 July 2009.

[3] Barhydt, R., Eischeid, T.M., Palmer, M.T., Wing, D.J.: Use of a prototype airborne separation assurance system for resolving near-term conflicts during autonomous aircraft operations. In: Proceedings of the AIAA Guidance, Navigation and Control Conference, Austin, TX, USA, AIAA (2003).

[4] FAA, 14 CFR Part 91, Automatic Dependent Surveillance-Broadcast (ADS-B) Out Performance Requirements To Support Air Traffic Control (ATC) Service, Federal Aviation Administration, 2010.

[5] DO-242A, Minimum Aviation System Performance Standards for Automatic Dependent Surveillance Broadcast (ADS-B), RTCA Inc, 2002.

[6] T.W. Rand and M.S. Eby. Algorithms for airborne conflict detection, prevention, and resolution. The 23rd Digital Avionics Systems Conference, October 2004.

[7] W. W. Y. Chung, D. Linse, O. Alaverdi, C. Ifarragurrie, D. Salvano and S. Seifert, "Surveillance and data link communication performance analysis for NextGen," 2012 Integrated Communications, Navigation and Surveillance Conference, Herndon, VA, 2012

[8] ICAO, DOC 4444, Air Traffic Management, Fourteenth Edition, 2001.

[9] Bilimoria, K.; and Lee, H.: Aircraft Conflict Resolution with an Arrival Time Constraint. Guidance, Navigation, and Control Conference, Monterey, CA, vol. AIAA 2002-4444, August 2002

[10] DO-317A, Minimum Operational Performance Standards for Aircraft Surveillance Applications, Washington; RTCA Inc, 2011.

[11] DO-289, Minimum Aviation performance stsnadards for aircraft surveillance applications (ASA), Washington: RTCA Inc, 2006. 
2017-12-04

\title{
Traffic avoidance and separation system
}

\author{
Katta, Balaji Raman \\ IEEE
}

Katta BR, Madani I, Traffic avoidance and separation system, Proceedings of 2017 International Conference on Advances in Computing, Communications and Informatics (ICACCI), 13-16

September 2017, Udupi, India

http://dx.doi.org/10.1109/ICACCI.2017.8126121

Downloaded from Cranfield Library Services E-Repository 